Mundo Agrario vol. 18, n 37, e047, abril 2017. ISSN 1515-5994

Universidad Nacional de La Plata.

Facultad de Humanidades y Ciencias de la Educación.

Centro de Historia Argentina y Americana

\title{
Solidaridad, Territorio y Coerción. Explicando la ausencia de conflicto en los trabajadores agroindustriales precarizados de la región del Maule, Chile
}

\author{
Solidarity, territory and coercion. Explaining absence of conflict in \\ the precarious agroindustrial workers, Region of Maule, Chile
}

Fernando Baeza Rivas *, Rodrigo Medel Sierralta **, Jorge Olea Peñaloza ***

* Centro de Investigación Político Social del Trabajo (CIPSTRA), ** Observatorio de Huelgas Laborales del Centro de Estudios de Conflicto y Cohesión Social (COES), *** Centro de Investigación Político Social del Trabajo (CIPSTRA). Departamento de Ciencias Históricas de la Universidad de Chile, Chile I f.baezarivas@gmail.com,medelcio@gmail.com,jorgeolea.p@gmail.com

\section{PALABRAS CLAVE}

Precariedad subjetiva

Conflictividad

Trabadores agroindustriales

Territorio

Chile

\section{KEYWORDS}

Subjective precariousness

Conflictivity

Agroindusrtial workers

Territory

Chile

\section{RESUMEN}

En base a 53 entrevistas realizadas a trabajadores agroindustriales de cuatro empresas de la región del Maule (Chile), se busca comprender las razones del desdoblamiento entre los indicadores objetivos de precariedad laboral y los bajos niveles de conflictividad en el agro, a partir del análisis de la insatisfacción subjetiva de los asalariados con su empleo. Esto se explicaría por factores territoriales, por el tradicionalismo de las relaciones laborales y por el miedo a sanciones de las empresas ante los conflictos. Los resultados enfatizan la necesidad de seguir profundizando en la precariedad subjetiva desde marcos conceptuales y metodológicos que enfaticen la trayectoria biográfica y laboral de los trabajadores.

Cita sugerida: Baeza Rivas, F., Medel Sierralta, R. y Olea Peñaloza, J. (2017). Solidaridad, Territorio y Coerción. Explicando la ausencia de conflicto en los trabajadores agroindustriales precarizados de la región del Maule, Chile. Mundo Agrario, 18(37), e047. 


\section{Introducción}

Una de las principales características del mercado laboral chileno es la precariedad de las condiciones de empleo (Narbona, Páez y Tonelli, 2011): los contratos temporales e inestables, la falta de acceso a seguridad social y los bajos niveles de ingresos, entre otros indicadores tradicionales, se han extendido como norma en amplios segmentos de la fuerza de trabajo.

Un sector donde la precariedad laboral es particularmente alta es el de los trabajadores agrícolas. En conjunto con la construcción y el sector de servicios, el sector agrícola es la rama donde más predominan los trabajos precarios, y los empleos protegidos apenas rondan el 20\% del total de trabajadores ocupados (Fundación SOL, 2015). En efecto, empleos con contrato formal indefinido, liquidación de sueldo, y cotizaciones previsionales de pensión y salud son una rareza en el agro chileno.

En contra de lo que pudiera imaginarse, dado lo extendido de la precariedad, la organización del colectivo de asalariados agrícolas es una de las más bajas del país, llegando la tasa de sindicalización de la rama apenas al 4,7\% durante el 2014 (último año para el cual hay registro a la fecha de esta publicación) ${ }^{1}$. Durante el mismo año, sólo se registraron cinco huelgas legales, y ajustando el total de huelgas (legales o ilegales) por la cantidad de trabajadores en la rama económica, se observa que el sector agropecuario es el que registra la menor conflictividad organizada de toda la fuerza de trabajo chilena, y que otros sectores con niveles similares de precariedad, como servicios y construcción, superan en 4 y casi 5 veces respectivamente los niveles de conflictividad del sector en cuestión (Pérez, Medel y Velásquez, 2015).

Ante esta desproporción surgen las preguntas que motivan la investigación cuyos resultados se presentan a continuación: ¿Por qué el sector con mayor precariedad en sus condiciones de trabajo resulta ser a la vez el sector con menores niveles de conflictividad laboral? Esta precariedad medida a través de los indicadores objetivos tradicionales, ¿'se traduce en un malestar subjetivo en los trabajadores agrícolas? Y si es así, ¿qué mecanismos operan para evitar que ese malestar se traduzca en conflictividad laboral organizada?

Para avanzar en respuestas, se retoman los aportes del sociólogo uruguayo Diego Piñeiro (2008; 2011), quien se propuso estudiar los efectos subjetivos de esta masiva precarización en la agricultura desde las definiciones de Paugam (2000), quien señala que para definir un trabajo como precario es preciso referirse a la doble vertiente de la precariedad: la objetiva, referida a las condiciones del empleo, y la subjetiva, que refiere a la relación entre el trabajador y su trabajo. En concreto, "será precario aquel que se encuentra en una relación precaria con su empleo y/o también aquel que sienta que su relación con el trabajo es insatisfactoria (...) ser precario será una combinación de sentir la precariedad y estar en la precariedad” (Piñeiro, 2008, p.14). Entre las condiciones objetivas y la percepción subjetiva de la precariedad no necesariamente existe una relación mecánica ni homogénea, y la forma en que el trabajador internaliza sus condiciones de trabajo dependen tanto del trabajo como de otras dimensiones no necesariamente ligadas al empleo actual.

El presente artículo busca comprender las razones del desdoblamiento entre los indicadores objetivos de precariedad laboral y los bajos niveles de conflictividad en el agro, a partir del análisis de la (in)satisfacción subjetiva de los asalariados con su empleo ${ }^{2}$. Así, se busca determinar si esta doble vertiente de la precariedad en el agro chileno puede servir para explicar la ausencia de conflictividad laboral en él. Para responder a este objetivo, se realizó una investigación cualitativa en la cual se entrevistaron a 53 trabajadores agroindustriales de cuatro empresas de dos territorios de la región del Maule, Chile.

A partir del análisis cualitativo de las entrevistas, se propone la existencia de tres mecanismos que permitirían gestionar el malestar subjetivo de los trabajadores y evitar una conflictividad que permanece latente y sin vías de escape: la solidaridad, el territorio y la coerción. 


\section{Precariedad laboral objetiva y subjetiva en el trabajo agrario-rural}

\section{La nueva ruralidad y la consolidación del precariado rural}

La mayor precariedad laboral de los sectores agrícolas se explica tanto por fenómenos naturales (variaciones estacionales y fluctuaciones climáticas en general) como por una serie de cambios producidos en el plano económico y social que han acentuado este fenómeno las últimas décadas. El difundido concepto de nueva ruralidad, alude precisamente a los cambios ocurridos en el campo a nivel latinoamericano como consecuencia de la ampliación de las relaciones de mercado hacia sectores rurales, buscando la integración del agro a la economía mundial, tendencia común en los países latinoamericanos volcados a la producción primaria (Pérez, Farah y C. de Grammont, 2008).

La consecuencia transversal de esto ha sido la disolución de las formas tradicionales de trabajo y producción típicas de los productores más pequeños y la consolidación del establecimiento de industrias orientadas hacia el exterior, que no solamente reposicionan la agricultura del continente en un nuevo lugar en el contexto internacional, sino que además introducen nuevas formas de explotación y organización de la fuerza de trabajo (Canales, 2005; Gómez, 2011; C. de Grammont, 2004 y Pérez; Farah y C. de Grammont, 2008). Algunas de las características predominantes actuales del trabajo rural son la pluriinserción, la flexibilización y la multiocupación a la que se ven forzados los habitantes de los territorios agrario-rurales en un contexto de crisis de la producción familiar (Bendini, 2008).

Si bien el aumento de la precariedad laboral no es un fenómeno exclusivo de los sectores agrario-rurales, sino de todo el modelo de desarrollo neoliberal, el sector en cuestión tiene particularidades que justifican un análisis por separado. Sumado a las condicionantes técnicas y climáticas de la producción agrícola, se tiene que la expansión agroindustrial en el campo chileno y latinoamericano en el período de instalación del neoliberalismo se constituyó sobre la base de pautas de subordinación y exclusión históricas que posibilitaban una estabilización de la precariedad de manera más masiva y abrupta que en otros sectores (Gómez y Klein, 1993 y Bendini, 2008).

En el caso chileno, la precariedad laboral del sector se ha evidenciado en innumerables estudios a partir de los datos de encuestas nacionales (Betancor, Modrego y Berdegué, 2008; Cid, 2001 y Reardon, Berdegué, y Escobar, 2004), sin embargo, poco se ha investigado sobre la precariedad o insatisfacción subjetiva que podrían percibir los trabajadores a partir de la forma en que internalizan las condiciones de empleo, elemento que hipotéticamente sería uno de los factores determinantes de la acción colectiva (Piñeiro, 2011, p. 30).

\section{Desacople entre precariedad objetiva y subjetiva en la nueva ruralidad}

Lo que han observado diversos investigadores en la ruralidad latinoamericana (Carámbula, 2009; Menéndez, 2009 y Piñeiro, 2011), a partir de la adopción más o menos laxa de la tipología de Serge Paugam, es un desacople entre las dos formas de la precariedad, o entre sus dos fuentes: protección y reconocimiento. Paugam señala que, cuando se rompe el vínculo laboral por la falta de protección, se pasa a formas de vinculación con el empleo ocasionales, con trabajos inestables y períodos de desempleo de larga duración; por su parte, cuando lo que se pierde es el reconocimiento, lo que aparece es la humillación social y el sentimiento de inutilidad. Por eso es que "el asalariado es precario cuando su empleo es inseguro y no puede prever su futuro profesional (...) pero el asalariado es igualmente precario cuando le parece que su trabajo no tiene interés y es débilmente reconocido en la empresa (...) siente ser más o menos inútil” (Paugam, 2000, 2012). A la combinación de ambas pérdidas o rupturas Paugam la denomina “descualificación social” (1991; Gaillie \& Paugam, 2000), siendo la forma paradigmática que puede adoptar la precariedad en los empleos generados en el neoliberalismo (Paugam, 2012). 
La condición de precariedad acarrea una serie de consecuencias sobre la vida de los trabajadores dentro y fuera del ámbito laboral. En efecto, los trabajadores precarios se encontrarían más expuestos a una situación de riesgo e inestabilidad laboral ( Guadarrama, Hualde y López, 2012 y Paugam \& Zhou, 2007), tanto por el riesgo de sufrir trastornos físicos -ya que cargan con los trabajos más pesados dentro de las faenas- como por las consecuencias psicológicas derivadas del estrés, la fuerte presión en el empleo y la constante incertidumbre respecto a la continuidad en el mismo. El riesgo social también se expresa en estos trabajadores en su condición de no integrados a los sistemas de protección social amparados en el derecho del trabajo para el resto de los asalariados (Castel, 2006).

Sin embargo, la presencia o ausencia de precariedad subjetiva no necesariamente deriva del riesgo social observado en las dimensiones objetivas de la precariedad. Si a nivel general la precariedad del trabajo surge como una pérdida o debilitamiento de la relación salarial en comparación con el modelo de desarrollo anterior -lo que permite identificar ciertas dimensiones objetivas a través de las cuales se puede clasificar a los trabajadores como más o menos precarios-, a nivel subjetivo e individual la interpretación de la situación de trabajo como precaria depende de cómo el trabajador vive esta pérdida. Así, por ejemplo, si para algunos trabajadores un empleo con contrato a plazo fijo representa un empleo precario, para otros puede resultar la única oportunidad para obtener ingresos y, por tanto, parecer un empleo favorable.

Precisamente, Bayón (2006) señala que el concepto mismo de empleo depende de un contexto espacial e histórico de estabilidad y formalidad que varía desde la comprensión del empleo que otorga protección e integración, al empleo que sólo entrega ingresos monetarios, y como en la población pobre la precariedad se concentra, la valoración del empleo allí tiende a estar más asociada a la obtención de ingresos que a la protección e integración social. Hipotéticamente entonces, se podría plantear que, si el estado de malestar asociado a la precariedad presupone la pérdida de los beneficios propios del empleo protegido, entonces difícilmente éste se haría presente a nivel subjetivo en los sectores de la población sistemáticamente excluidos que forman parte del precariado, sectores para los cuales nunca existió la promesa de la integración (Castel, 2010).

Por ejemplo, la trayectoria laboral de los trabajadores está muchas veces caracterizada por una constante alternancia entre empleo y desempleo, o una continuidad de empleos inestables, trayectoria que explicaría una cierta naturalización de la precariedad (Carámbula, 2009). De hecho, es frecuente que la carencia original de recursos básicos y habilidades útiles para el mercado laboral conduzca al entrampamiento de los individuos en oportunidades de vidas precarias, en las cuales las desventajas se retroalimentan y acumulan (Gaillie \& Paugam, 2000 y Bayón, 2006). Esta espiral de precariedad objetiva pone de manifiesto la lógica excluyente del modelo neoliberal, por cuanto genera grupos vulnerables a procesos de exclusión social (Pérez Sáinz, 2002-2003). No obstante, a nivel subjetivo podría operar inversamente, ocultando la precariedad por su naturalización en grupos de la población que sólo han conocido esta forma de empleo.

Dada la dinámica espacial del desarrollo capitalista rural latinoamericano, un factor que se torna especialmente relevante para el análisis combinado de la precariedad objetiva y subjetiva es el de la territorialidad. Diversos estudios (PNUD, 2008; Carámbula, 2009 y Canales y Canales, 2012) dan cuenta de la importancia que ha cobrado el "habitar territorial", ya sea en el sentido de las subjetividades asociadas a los territorios como en términos más concretos por la nueva vinculación con el trabajo y la ubicación geográfica de los centros productivos, tanto en el contexto general de las cadenas globales de comercialización, como sobretodo en el ámbito local de la movilidad espacial de los trabajadores dentro y fuera de los territorios donde habitan. En este sentido, deben distinguirse los conceptos de territorio y espacio, predominando para este análisis el primero por su mayor peso analítico, específicamente en lo referido a la idea de los puntos de conexión de los actores en los procesos de dinamización del espacio (Haesbaert, 2011), entre los que están precisamente las relaciones laborales. En otras palabras, no se trata 
sólo de espacializar cada uno de los elementos que van apareciendo en el análisis, sino de entenderlos dentro de la especificidad de cada lugar analizado, contextualizándolos y poniendo en perspectiva el peso de los procesos que ocurren en su interior.

Reconociendo entonces la extensión de la precariedad laboral en el sector, técnicamente necesaria para la expansión neoliberal en el campo, la pregunta por la insatisfacción de los trabajadores agroindustriales como fuente de conflictividad -o su contraparte, la sensación de satisfacción como explicación de la ausencia de conflictividad- cobra mayor relevancia y especificidad: ¿se encuentran los trabajadores agroindustriales, objetivamente precarios, insatisfechos con su trabajo? Los resultados obtenidos permiten reflexionar sobre las formas que adquiere la relación entre ambas formas de la precariedad, y luego sobre cómo esta relación basal puede servir para comprender la existencia de conflictos y el desarrollo de la acción colectiva entre los trabajadores rurales, o bien, la desintegración social dentro de este sector de los asalariados. En este sentido, el estudio apuesta por avanzar desde la descripción de la precariedad laboral en el sector hacia la comprensión de las consecuencias y repercusiones a nivel subjetivo en los actores individuales y colectivos.

\section{Metodología}

\section{Selección de los territorios y las empresas agroindustriales}

En Chile, la región del Maule es la que más ha crecido en el sector productivo de la agroindustria, aumentando progresivamente la intensidad de inversiones en la zona durante las últimas décadas. Estas nuevas inversiones están enfocadas en los sectores de mayor productividad y rentabilidad en la agroindustria -vitivinicultura, aceites de olivas, procesamiento de jugos, cultivos de berries en general- con ingresos autónomos más altos. Muchas de estas nuevas industrias están asociadas a innovaciones y alianzas generadas con los centros de estudios y universidades de la zona que tienen orientaciones hacia la agroindustria (ODEPA, 2014).

Este desarrollo es consecuencia del desplazamiento de cultivos y plantas de procesos desde regiones más septentrionales -O’Higgins y Metropolitana, principalmente- debido a la escasez hídrica en dichas regiones, lo que ha relocalizado ciertos cultivos más exigentes en el uso de este recurso, encontrando en la zona del Maule mejores condiciones sin alejarse demasiado de los centros de consumo y exportación más importantes (Muñoz y Navarro, 2011). Otro factor de relevancia que explica este desplazamiento de la agroindustria hacia la región del Maule es el menor valor de los suelos, que llega incluso a la mitad del de otras zonas comparables (ODEPA, 2009). En suma, es la región donde más se ha potenciado la agroindustria de manera sostenida en los últimos años.

Así, se puede señalar a la región del Maule como un espacio representativo de las nuevas formas y trayectorias productivas y laborales en el agro, el cual expresa el reacomodo típico que han experimentado los territorios rurales donde la producción agroindustrial se ha vuelto predominante. Además, la región representa de buena manera las viejas y nuevas formas de habitar el espacio, desde la consolidación de áreas agrícolas marginales producto de la contrarreforma y los productores desfavorecidos por la redistribución de tierras (Bengoa, 1983), hasta el desplazamiento poblacional desde las zonas rurales hacia las ciudades intermedias de la región.

En este sentido, los procesos que van dando forma a estos espacios son analizados bajo la premisa de la territorialidad de las relaciones sociales, es decir, la identificación de las prácticas que inciden en la construcción social de estos territorios, y en cómo estas repercuten en la conformación de dichas relaciones sociales (Sack, 1986). 
Pese al dinamismo de casi toda la región durante las últimas décadas, dentro de ella coexisten territorios claramente diferenciables por el momento en que se insertan en el proceso de modernización neoliberal. Si en la zona de Talca el señalado dinamismo es cuestión de apenas algunos lustros, en el territorio de Curicó (70 kilómetros más al norte) la modernización neoliberal está lejos de ser reciente, y se encuentra, de hecho, consolidada. Por esta razón es que la muestra diferencia dentro de la región a los territorios de Curicó y Talca. Para la selección de casos dentro de cada territorio se procedió, en primer lugar, a distinguir las comunas agrario-rurales, prototípicas de la nueva ruralidad y donde el sector agrario es el preponderante de entre todas las ramas económicas $\underline{3}$. Así, de las 30 comunas de la región, 11 fueron clasificadas en este grupo. Luego, se distinguieron las comunas agrario-rurales que pertenecían a cada territorio (Curicó y Talca), para finalmente seleccionar 4 unidades productivas ubicadas en comunas diferentes (Figura 1), que desarrollaran procesos industriales similares y que correspondieran a los rubros predominantes en el modelo agroindustrial que se ha impuesto en la región.

\section{Tabla 1}

\begin{tabular}{|l|l|c|c|c|}
\hline \multicolumn{5}{|c|}{ Comunas, porcentaje de ocupación en actividades silvoagropecuarias, empresas seleccionadas y sus } \\
rubros \\
\hline Territorio & Comuna & $\begin{array}{c}\text { \% de la fuerza de trabajo } \\
\text { en actividades } \\
\text { silvoagropecuarias } \\
\text { (CASEN 2011) }\end{array}$ & Empresa seleccionada & Rubro \\
\hline Talca & Pelarco & 58,4 & Almazaras del Pacífico & Aceites \\
\hline & Pencahue & 51,8 & Las Doscientas & Aceites \\
\hline Curicó & Romeral & 65,1 & Copefrut & $\begin{array}{c}\text { Congelados; } \\
\text { Deshidradatos }\end{array}$ \\
\hline & Teno & 55,8 & Agrícola Forestal el Escudo & Jugos \\
\hline
\end{tabular}

Fuente: elaboración propia en base a datos de la encuesta de Caracterización Socioeconómica Nacional (CASEN) 2011 y del Directorio de la Agroindustria Hortofrutícola Chilena 2011.

\section{Producción de información}

Para llevar a cabo este estudio se adoptó un método cualitativo basado en entrevistas semiestructuradas que tenían el objetivo de profundizar en los mecanismos que generan acople o desacople entre las dimensiones objetivas y subjetivas de la precariedad laboral. Previo a la realización de las entrevistas, se aplicó un breve cuestionario para lograr una mejor caracterización y clasificación de los casos según atributos en variables tanto sociodemográficas como de condiciones laborales, satisfacción con el empleo y organización colectiva. La pauta de entrevista, por su parte, se estructuró en base a siete grandes temas: caracterización general de los trabajadores; caracterización general del empleo y precariedad objetiva; identificación de posiciones laborales y diferenciación interna de los trabajadores; migración laboral; precarización subjetiva; organización sindical; conflictos laborales y disposición a la organización.

El trabajo en terreno se realizó entre los meses de abril y junio del año 2014, entrevistando en total a 53 trabajadores involucrados directamente en las faenas productivas, 25 de modo permanente (durante todo el año) y 28 como temporeros o por periodos definidos. La distribución también fue equitativa entre territorios, aun cuando en algunas empresas se realizaron más entrevistas de las originalmente planificadas, como en AFE (Tabla 2). Para la selección de los trabajadores se tuvo en consideración variables como sexo, edad, 
nivel educacional y ocupación, con el objetivo de analizar la realidad de trabajadores en distintos niveles de precariedad objetiva, así como la diversidad de subjetividades presentes (Dávila, 1995).

Tabla 2

\begin{tabular}{|l|l|c|c|}
\hline \multicolumn{4}{|c|}{ Muestra por empresas y territorio } \\
\hline \multicolumn{1}{|c|}{ Territorio } & \multicolumn{1}{|c|}{ Empresa } & Frecuencia & \% \\
\hline \multirow{2}{*}{ Curicó } & Copefrut & 10 & 18,9 \\
\cline { 2 - 3 } & AFE & 17 & 32,1 \\
\hline \multirow{2}{*}{ Talca } & Almazaras del Pacífico & 12 & 22,6 \\
\hline Total & Las Doscientas & 14 & 26,4 \\
\hline
\end{tabular}

Fuente: Elaboración propia

\section{Análisis de la información}

Una vez transcritas, las entrevistas fueron analizadas con el software NVIVO 8, asignándole a cada entrevistado los atributos de caracterización señalados en el cuestionario inicial. Para el análisis de la información se utilizó la metodología de teoría fundamentada (Glaser \& Strauss, 1967 y Glaser, 1992), en función de elaborar hipótesis y encontrar dimensiones que explicaran los posibles acoples y desacoples entre la precariedad objetiva y la precariedad subjetiva, así como la relación de ambas con la conflictividad laboral. Tras una primera lectura general de las transcripciones, la codificación se realizó en tres etapas sucesivas: codificación abierta, donde se identificaron las tres dimensiones en que se desarrollan los resultados; codificación axial, donde se construyeron las relaciones internas a las dimensiones o grandes códigos (por ejemplo, la distinción de la solidaridad entre trabajadores y la solidaridad entre la empresa y los trabajadores); y codificación selectiva, donde se retomó el marco teórico para enriquecer los conceptos construidos.

Para determinar la precariedad objetiva, se construyó un “índice de protección del empleo” el cual incorpora algunos de los elementos más básicos que la literatura clásica sobre la materia recomienda incluir para la medición de la precariedad y/o la protección del empleo tanto a nivel nacional (Fundación SOL, 2015) como internacional (Rodgers \& Rodgers, 1989; ESOPE, 2005 y Piñeiro, 2011), enfatizando los componentes más relevantes para los objetivos de la investigación. Cuatro indicadores dicotómicos se sumaron linealmente para el cálculo del índice: la presencia de contrato, la duración indefinida del contrato ${ }^{4}$, la existencia de instancias de negociación al interior de la empresa y que los ingresos mensuales del trabajo superaran el ingreso mínimo legal mensual (para el año 2014 en Chile, \$210.000, unos US\$400). De esta forma, cada entrevistado podía obtener un puntaje de entre 0 y 4 puntos en el índice. Para facilitar la clasificación de los casos, estos puntajes se reagruparon en tres categorías según el nivel de protección del empleo: baja (0 a 2 puntos), media (3 puntos) y alta (4 puntos).

A modo exploratorio y con el único objetivo de clasificar a los entrevistados para el análisis de la información, se incluyó en el cuestionario inicial una pregunta ordinal sobre el nivel de satisfacción con el empleo, pudiendo responder cada entrevistado que se encontraba "muy satisfecho", "medianamente satisfecho", "ni satisfecho ni insatisfecho" o "insatisfecho". Como era de esperar, la mayor parte de los entrevistados señaló sentirse "muy satisfecho" con su empleo, por lo que para el análisis se distinguen dicotómicamente las respuestas entre "satisfechos" (los que respondieron "muy satisfechos”) y "algo 
insatisfechos” (los que señalaron cualquier otra respuesta).

Figura 1. Mapa de Chile, de la Región del Maule y distribución de las empresas analizadas

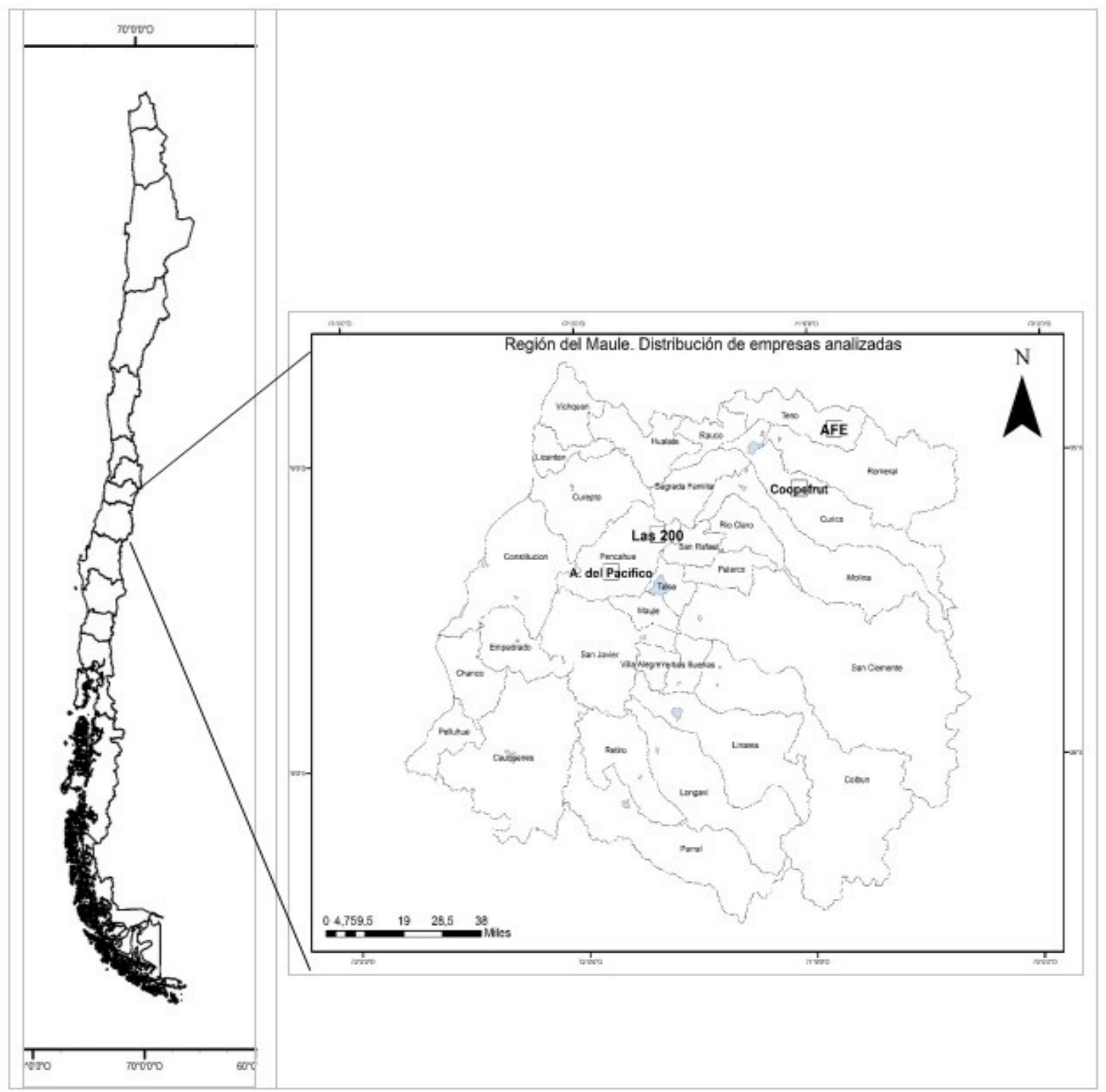

Fuente: elaboración propia

\section{Caracterización de la muestra}

Como se observa en la Tabla 3, la muestra se compone principalmente por hombres, trabajadores de entre 20 y 60 años y sin educación superior, en línea con lo observado a nivel nacional entre los asalariados agroindustriales. 
Tabla 3

\begin{tabular}{|c|c|c|}
\hline \multicolumn{3}{|c|}{ Caracterización sociodemográfica de los entrevistado } \\
\hline Variables & Categorías & Frecuencia \\
\hline \multirow{2}{*}{ Sexo } & Masculino & 35 \\
\hline & Femenino & 18 \\
\hline \multirow{6}{*}{ Grupo etario } & Menos de 20 años & 2 \\
\hline & 20-29 años & 11 \\
\hline & 30-39 años & 15 \\
\hline & 40-49 años & 12 \\
\hline & 50-59 años & 10 \\
\hline & 60-69 años & 3 \\
\hline \multirow{3}{*}{$\begin{array}{l}\text { Nivel } \\
\text { educacional }\end{array}$} & Básica & 24 \\
\hline & Media & 20 \\
\hline & Técnico superior & 7 \\
\hline \multicolumn{2}{|c|}{ Total (incluye perdidos) } & 53 \\
\hline
\end{tabular}

Fuente: elaboración propia

Es importante señalar que en ninguna de estas cuatro empresas agroindustriales se constató presencia de sindicatos, o de cualquier forma de organización de trabajadores. Esto se condice con los antecedentes discutidos en las secciones anteriores, que hablan de un bajo nivel de conflictividad y organización sindical en el agro chileno.

Por su parte, considerando los casos que registran todas las respuestas válidas para la construcción del índice de protección del empleo, se observa que el 51\% de los entrevistados registra niveles bajos de protección del empleo, el 32,7\% niveles medios y el 16,3\% una alta protección del empleo. Al contrastar las respuestas a la pregunta por la satisfacción con el empleo por el nivel de protección en el mismo, se observa que ambas formas de la precariedad no van de la mano (Figura 2) y que en las empresas analizadas parece ocurrir lo mismo que en el caso uruguayo (Piñeiro, 2011), donde los sectores rurales caracterizados por una alta precariedad laboral objetiva no manifiestan niveles similares de insatisfacción subjetiva. En efecto, la forma en que se distribuyen los niveles de satisfacción no varían significativamente en cada nivel de protección del empleo, siendo siempre mayoritario el grupo que señala encontrarse "satisfecho", independientemente del nivel de precariedad objetiva del empleo ${ }^{\underline{5}}$. 
Figura 2: Satisfacción con el empleo según índice de protección del empleo

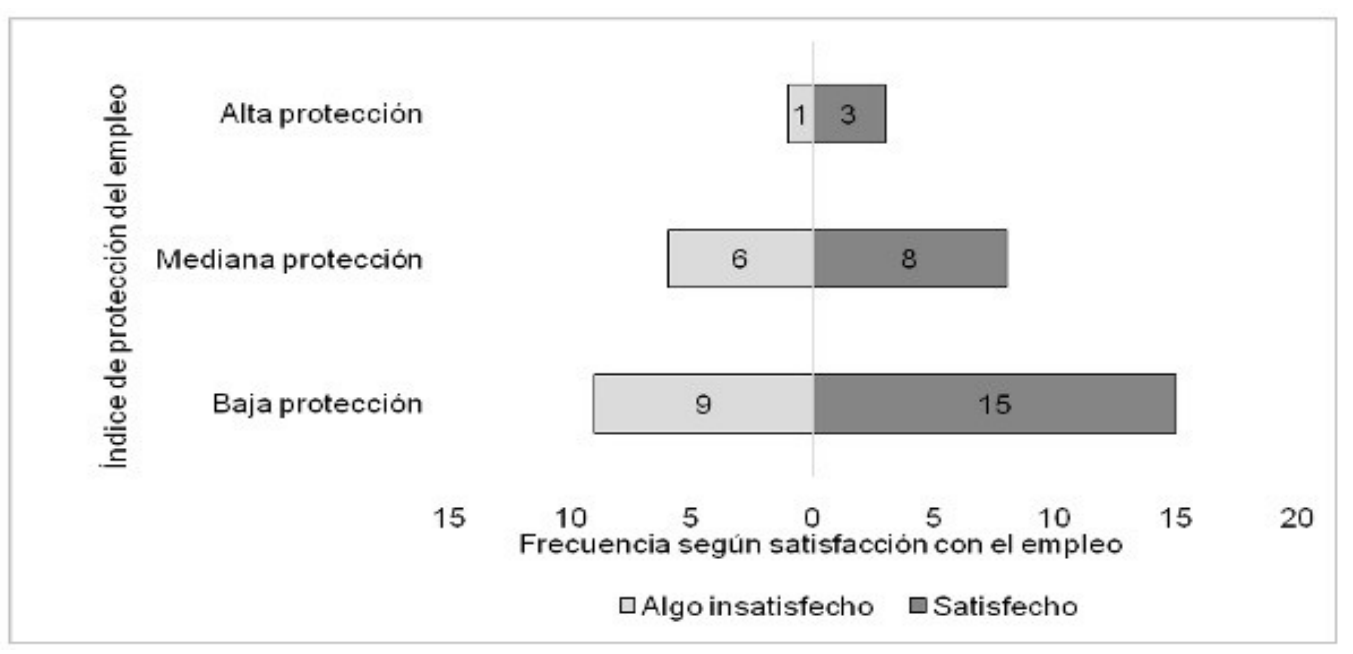

Fuente: elaboración propia

\section{Explicando la brecha entre estar en la precariedad y sentirse precario}

La Figura 2 permite adentrarse en los principales resultados obtenidos a partir de las entrevistas, lo que ayuda a interpretar parte de la tensión entre "ser y sentirse precario", al decir de Piñeiro (2011), la cual ha sido identificada por diversos autores, pero no explicada con mayor profundidad. A partir de la investigación realizada y del análisis de la información cualitativa, puede darse cuenta de que entre los trabajadores agrario-rurales de la región del Maule operarían tres mecanismos que explican la ausencia de insatisfacción y, conjuntamente, también la inexistencia de conflictos laborales manifiestos: la sociabilidad y cotidianidad propia del territorio, que generan la sensación de encontrarse en ambientes de trabajo favorables; la solidaridad, como un valor destacable y rescatable por los trabajadores; y finalmente, las estrategias coercitivas de la empresa, que actúan como un impulso a delimitar las aspiraciones de los trabajadores, tanto por obtener un mejor empleo como por la posibilidad de organizarse.

\section{Sociabilidad y cotidianidad}

Este mecanismo se encuentra frecuentemente en las entrevistas, asociado a la idea de mantener un buen ambiente de trabajo, en donde no haya conflictos ni desavenencias que generen un sentimiento de competencia entre los trabajadores. En ese sentido, se plantea que un buen ambiente se lograría manteniendo una cotidianidad en donde todos deben participar: los jefes no exigiendo excesivamente, los trabajadores no perdiendo el tiempo -no "conversando tanto"-, en fin, todos cooperando en la mantención de un buen ambiente y productividad.

Otro de los principales elementos evaluados positivamente para mantener una buena convivencia es poder trabajar con personas conocidas, con vecinos: “Sí, la mayoría de la gente que trabaja acá son de acá, son pura gente casi pura gente conocida. Si la gente que no es conocida son los que trabajan por contratistas” (AFE, trabajadora). El sentido de pertenencia a un lugar otorga un respaldo a las buenas relaciones que se establecen con los demás trabajadores, ya que las trayectorias de vida en conjunto funcionan tanto en el ámbito del trabajo como en la vida cotidiana. Así, el reconocimiento de un territorio común -esta vez no Talca ni Curicó, sino las localidades, pueblos y pequeñas ciudades donde viven la mayor parte de los trabajadores de cada empresa- y las prácticas que sustentan dicha territorialidad, serían claves para entender las relaciones que explican la valoración positiva otorgada al trabajo, en cuanto espacio común donde se vive 
el territorio.

La cotidianidad que caracteriza al trabajo y a las relaciones dentro de este espacio, se expresa también en el arraigo de los trabajadores a un territorio, que termina siendo en algunos casos una territorialización forzada. Por ejemplo, la empresa AFE se instaló en la localidad de El Escudo y otorgó una serie de regalías a los trabajadores relacionados en general con la adquisición de casas hace un par de décadas. Por otra parte, la localidad de El Escudo se ubica en el valle del Huemul, comuna de Teno (al noreste de Curicó), con una difícil conexión a la principal vía, la carretera 5-Sur, cuestión que dificulta enormemente la posibilidad de acceder a otros empleos sin incurrir necesariamente en una inversión significativa de tiempo y, sobre todo, dinero. Por esto, prácticamente todos los habitantes de El Escudo trabajan en AFE, situación que se repite en los entrevistados de otras empresas que sólo por incrementos salariales significativos justificarían un cambio de empleo a un lugar de trabajo más alejado, aplicando una estricta racionalidad económica, como se observa en las palabras de un joven temporero de 18 años:

Acá la gente tiene miedo, porque hay personas que piensan que el Escudo es su fuente laboral, por ejemplo ‘no, si me voy de acá, ¿para dónde voy a ir? Es lo único que sé hacer’. Entonces, igual, a mí me han ofrecido varios tipos de trabajo, pero el tema es que a lo mejor también puedo pensar 'si me voy del Escudo me voy a morir de hambre’, pero no es así, yo tengo pega, pero ya el tema que, si yo acá gano los 300, puedo ganar 350, 400 en otro lado, pero ya tengo que empezar a pensar que tengo que pagar el doble de agua que pago, el doble de la luz, y tengo que pagar el arriendo, y tengo que pagar locomoción. (AFE, trabajador)

En una situación similar de lejanía respecto a los principales centros urbanos se encuentran algunos trabajadores de la empresa Almazaras del Pacífico en la comuna de San Rafael (al norte de Talca), especialmente los que viven en el sector de Alto Pangue. En este caso, la ciudad de referencia es Talca, cabecera regional y foco de la emigración laboral, pero que para estos trabajadores se presenta mucho más lejana, debido entre otras cosas a cuestiones familiares como las que presentaba un trabajador de 37 años, quien reflexionaba: "he pensado en cambiarme... de repente me gustaría, pero por el colegio de los niños no, está cerca” (Almazaras del Pacífico, trabajador).

Esta situación muestra una profunda contradicción entre la modernización de las formas productivas y las relaciones laborales, ya que en muchos de estos casos la adscripción al territorio está determinada por la estrecha dependencia laboral de estas empresas y la falta de alternativas. Esta situación de concentración de lo laboral y lo cotidiano en un solo territorio, provoca que la necesidad de mantener buenas relaciones entre los trabajadores (que también son vecinos, amigos o familiares) realcen las formas tradicionales de vinculación social en el espacio de trabajo.

Por otra parte, la decisión de dónde trabajar está contenida por factores de desempeño laboral, donde la reflexión cruza la evaluación de las aptitudes que posee cada trabajador, lo que va asociado a una "cultura del trabajo", a las formas y relaciones que se entablan a partir de la labor que se desempeña, es decir a una forma estática de entender la condición de trabajador:

Yo por lo menos no encontraría [trabajo] en una tienda por decirte, no sabría cómo desempeñarme, me costaría montones. Pero si yo me fuera de una empresa a otra, a un taller, me sería mucho más fácil enrolarme al tiro, porque uno ya tiene conocimiento, y de la maquinaria de un lado a otro, es poca la diferencia (Copefrut, trabajador).

\section{Solidaridad}

Otro aspecto clave para entender el desencuentro entre la precariedad objetiva y la subjetiva se encuentra 
aldimensionar los aspectos concernientes a las relaciones de apoyo y compromiso mutuo, las cuales son altamente valoradas en las relaciones laborales cotidianas. En este sentido habría que hacer una distinción entre dos niveles de solidaridad: la de trabajador a trabajador, y la que se da entre la empresa y el trabajador. En ambos casos se trata de un elemento que es frecuentemente señalado como un mecanismo a través del cual se resuelven los conflictos entre los propios trabajadores y, asimismo, con la empresa, siendo en el ámbito individual y de trato directo donde se solucionan la mayoría de las diferencias, volviéndose aparentemente innecesario algún tipo de asociación. En otro nivel, funciona como una especie de indicador de un buen trabajador y compañero, el cual portaría esa capacidad de solucionar rápida y eficazmente cualquier tipo de conflicto, situación que es transversal a casi todas las empresas.

\section{Solidaridad entre trabajadores}

Uno de los elementos que más resalta es una solidaridad de vocación individual, es decir, que la solidaridad se entiende como un mecanismo que surge en momentos difíciles (alguna tragedia personal o familiar) de un individuo y que articula el apoyo de otros trabajadores:

Somos solidarios, y esa es otra cosa, que nosotros siempre alegamos porque, por ejemplo, se enferma alguien o le pasa algo malo a alguien, siempre 'oye, hagamos una colecta', 'ya, de 5 lucas' [5.000 pesos chilenos, unos US\$8], y ya, ahí llegan, que si vamos a hablar, o alguien habla que si se nos puede descontar por caja. Entonces ya, se nos descuenta por caja, pero el año, cuando fue el terremoto [2010], ahí cada trabajador aportó con un saco de carbón para los más afectados (AFE, trabajadora).

Esto suple de cierta manera la necesidad de exigir a la empresa una mayor acción formal o protección, y se reemplaza por una solidaridad que toma la forma de una "campaña solidaria”, suplantando las prácticas formales contempladas en la legislación vigente:

En el proceso, igual en el packing, también se organiza nuestra gente, también tienen su propio bienestar, ellos se ayudan. Ahí sí que uno ve la solidaridad, ahí la gente de temporada, hay una persona que se enferma y todo el mundo está ayudándolo haciéndole rifa, haciéndole colecta. Es muy común ese tema de 'andamos haciendo una colecta para tal señor que le pasó esto, esto otro'. Sí, la gente ayuda mucho (Copefrut, trabajadora).

Estas manifestaciones de apoyo mutuo son vistas positivamente, ya que expresarían de cierto modo el compromiso e identificación de los trabajadores con los pares, procedimiento que se encuentra ya consolidado en los diversos sectores analizados en esta investigación.

\section{Solidaridad desde la empresa}

Esta se presenta de la misma manera que en el ítem anterior, en cuanto a que se espera que la empresa actúe en los momentos de situaciones extraordinarias de sus trabajadores. Según los testimonios recogidos, al estar vinculados por medio del trabajo, la empresa debería aplicar los mismos principios que entre los trabajadores, aunque no de manera formal ni institucionalizada, sino que reactiva ante los problemas que puedan ir surgiendo en las vidas de los trabajadores. Esto establece una posición de agradecimiento con respecto a la empresa desde los trabajadores, ya que, al no estar sancionada en los instrumentos oficiales, sería una acción motivada por las buenas prácticas de los jefes y patrones:

Igual nos han ayudado con monedas [dinero], para ir a ver a mi hija al hospital, porque igual ella estuvo harto tiempo en el hospital. Entonces igual esas cosas uno no las espera de los patrones (...) 
uno no espera de alguien que lo ayude así, pero así lo hicieron (AFE, trabajadora).

$\mathrm{Y}$ al igual que en el caso de trabajador a trabajador, la ausencia de esta solidaridad es presentada como algo que estaría en contra de esta especie de "mandato ético":

Incluso a nivel empresa, la empresa me hubiese dicho 'chuta Manuel, mi más sentido pésame', y un arreglo floral. Nada. Nada de nada. Entonces, ahí de repente hay momentos en que la empresa flaquea, pero igual de repente son casos aislados. Y de repente la culpa la tiene el jefe de área que no da a conocer, pero hay cosas que yo creo que hacemos vista ciega a la realidad (AFE, trabajador).

Incluso existen casos donde se ha evidenciado la combinación de estas solidaridades, tanto desde los propios trabajadores como desde la empresa, atendiendo necesidades materiales y de acompañamiento en las labores de cobijo y contención:

Nosotros hemos tenido casos de gente que se les ha quemado sus casas, y cuadrillas completas de aquí incluyendo a la empresa han salido y han ayudado a la colaboración, ya sea en la remoción de escombros, en la reinstalación de la nueva casa, no sé lo que sea, pero siempre hay personas. Un enfermo necesita recursos, se hace una colecta y se le junta plata y se le entrega a él o a la familia. El tema de los funerales siempre hay gente que va y acompaña a los deudos, hay una preocupación por el otro compañero (Copefrut, trabajador).

Las relaciones de solidaridad entre trabajadores y de los trabajadores con la empresa, son relaciones no pactadas que se consolidan como una solución efectiva, pero contingente y pasajera, sin necesidad de asociaciones estables y formales entre los trabajadores y prácticas legales e institucionalizadas de protección y bienestar desde la empresa.

\section{Coerción de la empresa}

Uno de los puntos más complejos está situado en las tensiones laborales no explicitadas en la cotidianidad, pero que los trabajadores saben que existen: listas negras que impiden que trabajadores más conflictivos sean reincorporados o incluso contratados en otras empresas del territorio; castigos fuera de la ley; no opinar para no ser castigado; engaños en el sistema de pago; y vacíos por la ausencia o disfuncionalidad del sindicato. La coerción de la empresa, que opera principalmente a través del temor a las represalias, genera en los trabajadores, al mismo tiempo, una invisibilización de la precariedad objetiva y la falta de organización colectiva.

Las prácticas coercitivas de las empresas representan un aspecto clave en la triada precariedad objetiva, precariedad subjetiva y conflictividad, ya que es el elemento por donde la insatisfacción aparece con más claridad y, por lo tanto, donde la sensación de la precariedad subjetiva comienza a tomar forma, ya sea relativizando los puntos anteriores, que son considerados positivamente, como evidenciando la precariedad objetiva de los trabajadores dentro de la empresa.

Los registros de trabajadores "problemáticos" son una generalidad para las empresas de los territorios estudiados. Para este fenómeno existiría una explicación desde los trabajadores y otra desde la administración de personal, lo que da cuenta de que la apreciación de la conflictividad varía según la posición que se ocupe en la estructura laboral. Por ejemplo, una trabajadora de planta de AFE señala no sólo haber oído de la aplicación de castigos, como la expulsión del lugar de trabajo, o el desarrollo de labores ajenas al trabajo pactado, con la consecuente merma en la parte del salario que se obtiene por producto, sino también conocerlos de primera fuente: "porque incluso mi esposo una vez lo castigaron: que se tenía que ir a trabajar a Candelaria, que Candelaria queda en la punta no sé dónde, para allá” (AFE, trabajadora). 
En la empresa Copefrut se darían situaciones aún más extremas, como que, al primer reclamo de los trabajadores, estos son inmediatamente despedidos. Así lo señala una trabajadora temporera:

Si tú reclamas un poquito por algo sabes que chao, te fuiste, ya nada. Muchas veces sin siquiera un aviso ni te dicen 'conversemos lo que está pasando', nada. Te vas sin previo aviso (...) cuando están embarazadas las hacen firmar unos papeles obligadas, como para renunciar al trabajo y echarlas (Copefrut, trabajadora).

En las empresas en que se han desarrollado prácticas contra los trabajadores existen, al mismo tiempo, mecanismos que limitan la posibilidad de llevar a cabo algún tipo de conflicto legal al interior de las plantas y faenas. Por ejemplo, en la empresa AFE, quien intenta interponer un reclamo es directamente despedido, razón por la cual una trabajadora señala que hay un ambiente que no permite realizar ningún tipo de protesta o reclamo formal: "Por eso nosotros cuando están ellos por ahí, los jefes más grandes o los patrones, nosotros preferimos no comentar esas cosas políticas o algo así, o andar liderando cosas, porque si no, capaz que nos echen" (AFE, trabajadora).

Las veces que se ha intentado realizar alguna acción de protesta, la empresa toma medidas inmediatas, apelando a las ya mencionadas solidaridad y cotidianidad presentes en el espacio de trabajo:

No, si lo más malo ahí es que, pongámosle, si yo quiero hacer un paro [Huelga], hago el paro, pero se tiran al tiro encima los jefes de planta: 'Ya, sabes qué, a este para afuera'. A un amigo le pasó así. Hizo un paro para la temporada de la cereza, dijo: 'párense no más, no pueden ser así, a pedir aumento de sueldo’, y lo que pasó es que paró toda la gente, y ahí después al otro día le dieron puerta [despido] (Copefrut, trabajador).

La inmovilización que generan todas estas prácticas es evidente, ya que operan en las mismas redes que generan la precariedad. La desvinculación del trabajador con su trabajo está sostenida en que la única certeza de la movilización está asociada al despido, y no a la posibilidad de cambio de condiciones:

Yo creo que más que nada es exactamente lo mismo, porque a veces cuando ha habido temas que han pasado y conversan 'oye, ¿’por qué no hacemos esto, nos juntamos todos y hablamos con los jefes?', y todos dicen ‘¿para qué? Si no vamos a lograr nada, ¿para que nos echen?’. Es algo que ya todos tienen como clarito (Copefrut, trabajadora).

Esta situación de coerción permite también explicar la fractura entre estar en la precariedad y sentirse precario en el mundo rural, al sostener de manera activa ciertos dispositivos que mantienen los niveles de insatisfacción controlados, obligando ante la precariedad de los empleos a generar otras fuentes de contención de esos problemas, como la solidaridad y la sociabilidad. Al mismo tiempo, también permite explicar en parte por qué el sector agrícola presenta las más bajas tasas de sindicalización y conflictividad laboral, ya que la posibilidad de levantar organizaciones de trabajadores, por básicas que estas sean, se encuentra limitada desde su origen.

\section{Conclusiones}

El rol que hipotéticamente se le había asignado a la precariedad subjetiva para comprender la ausencia de conflictividad laboral en un sector con tan altos niveles de precariedad objetiva, resulta al mismo tiempo confirmado y matizado.

Si bien sólo 4 entrevistados declararon sentirse derechamente insatisfechos con su trabajo, no podría decirse que el resto de los trabajadores de las empresas analizadas no se encuentren también precarios subjetivamente. Lo cierto es que, si bien los entrevistados no señalaron explícitamente sentirse insatisfechos, 
al analizar con mayor profundidad sus relatos se encuentran señales claras de precariedad subjetiva. Es la falta de-explicitación del malestar lo que permitiría explicar la ausencia de conflictividad en estos trabajadores, porque no habría un motivo evidente, compartido y, sobretodo, socializado que justifique manifestar colectivamente el conflicto. Sin embargo, esto no significa que los trabajadores no reconozcan en las entrevistas un malestar más profundo, especialmente por lo que Paugam llamaría una falta de reconocimiento, típica de la situación de descualificación social (2012), evidenciada por ejemplo en las prácticas coercitivas de las empresas del sector en la región.

Así entonces, lo que a primera vista aparece como un desacople (entre la precariedad objetiva y la mayoritaria satisfacción con el empleo), expresa en realidad una precariedad subjetiva oculta, o implícita, cuya falta de manifestación anula la posibilidad de la generación de conflictos en el espacio de trabajo. Concretamente, se observan tres mecanismos que operarían consecutivamente anulando la explicitación de la precariedad subjetiva y, en consecuencia, del conflicto: la sociabilidad y cotidianidad del territorio, la alta densidad de las relaciones ejemplificadas a través de la solidaridad, y la coerción de la empresa.

Lo que en las entrevistas emerge como sociabilidad, expresa, en el fondo, dos dimensiones y escalas de lo territorial: por una parte, la importancia de las relaciones sociales cotidianas para generar un sentido de pertenencia y vivir el espacio de trabajo como un espacio del territorio; esto es, el territorio a pequeña escala, o derechamente el pueblo donde se habita. Por otra parte, la homogeneidad de oportunidades al interior del territorio, especialmente para quienes tienen menos recursos para insertarse provechosamente en el mercado ocupacional incluso en una escala mayor, en estos casos, las provincias de Talca y Curicó. Ambos elementos redundan en las bajas expectativas que manifiestan los entrevistados ante la posibilidad de tener una situación social y laboral mejor que la actual, tanto por las dificultades asociadas a salir del lugar como porque, en realidad, las alternativas ocupacionales no justifican un cambio ni, mucho menos, iniciar un conflicto. En definitiva, si bien este componente podría generar vínculos que potenciaran en el largo plazo la organización colectiva, por lo pronto lo que se observa es resignación.

El segundo mecanismo, la solidaridad entre trabajadores y de la empresa para con los trabajadores, expresa una particularidad de los espacios rurales que se repite al interior del ámbito del trabajo: las relaciones que allí se establecen son densas, informales, y las diferencias se resuelven individualmente. Obviamente, la falta de institucionalización de las relaciones por la efectividad de la solidaridad contingente, agudiza la diferencia estructural entre patrón y trabajador. La institucionalidad formal que regula las relaciones laborales en otros espacios, ausente en las empresas estudiadas, es reemplazada por un marco ético de informalidad y tradición por todos conocido y, en general, por todos respetado. Así, cuando los trabajadores llegan a sobrepasar la primera barrera, la de la resignación, y se enfrentan a la empresa, lo hacen individualmente, lógicamente con magros resultados, que en general tienden a consolidar la densidad de las relaciones tradicionales.

Pero aún hay un tercer mecanismo que se expresa cuando la resignación y la manifestación individual comienzan a ser superadas: la coerción de la empresa. Aquí lo que opera es el miedo a las represalias que, autoritariamente, pueda adoptar la empresa, incluyendo castigos, listas negras y despidos. De esta forma, todos saben que hay ciertos temas o reclamos que no se pueden expresar, o al menos no colectivamente, $\mathrm{y}$ que la disconformidad o insatisfacción no puede organizarse.

Independiente de si los trabajadores señalaron encontrarse satisfechos o insatisfechos con sus empleos, o del nivel de precariedad objetiva que cada uno presentara, estos tres elementos aparecieron de forma transversal en las entrevistas realizadas en las cuatro empresas analizadas. Difícilmente podría señalarse que la resignación a la inexistencia de un empleo mejor o el miedo al despido equivalgan a sentirse subjetivamente protegido. En este sentido, una conclusión del estudio es que es necesario adentrarse desde metodologías cualitativas apropiadas al contexto de cada territorio ya la realidad de los trabajadores rurales latinoamericanos, como así también al análisis de conceptos como precariedad subjetiva, insatisfacción o 
malestar. Como se pudo apreciar, la primera respuesta de los entrevistados al cuestionario de caracterización sobre la satisfacción con el empleo no reflejaba acertadamente la precariedad subjetiva descubierta en las entrevistas, lo que podría haber conducido a errores de interpretación que, por ejemplo, explicaran el desacople por las características "tradicionales” de estos trabajadores, como las tesis históricas que ven en este sector de los asalariados a los herederos de la "paz social" de la hacienda del valle central (Bengoa, 1988).

En este sentido, futuras investigaciones podrían indagar en los factores que influyen en declararse abiertamente insatisfecho con el empleo, ya que no toda precariedad subjetiva parece tan evidente a primera vista, ni siquiera para los propios trabajadores, lo que no significa que esta no exista. Consecuentemente, resulta fundamental utilizar más decididamente técnicas de producción de información que asuman estas dos manifestaciones de la precariedad subjetiva (explícita e implícita), como las entrevistas en profundidad y las etnografías.

Por otra parte, se debería investigar con mayor profundidad la percepción subjetiva de la precariedad dadas ciertas características sociodemográficas, ocupacionales, y sobretodo, biográficas de los trabajadores, ya que en las historias de vida de cada entrevistado aparecen otros elementos no considerados con mayor detalle en esta ocasión, los cuales podrían explicar las distintas percepciones de la precariedad en base a las distintas expectativas de empleo generadas, a su vez, por la trayectoria laboral de precariedad o protección (Carámbula, 2009 y Castel, 2010), cuestión especialmente relevante en estos sectores caracterizados por la pobreza, la escasez de capital educativo y de recursos básicos para el mercado laboral en general, que provocan un entrampamiento en la precariedad (Gaillie \& Paugam, 2000).

Finalmente, se puede concluir que uno de los grandes desafíos para los asalariados agrícolas está en avanzar hacia una mayor modernización de las relaciones laborales en las empresas del sector. Probablemente, romper con el tradicionalismo de la solidaridad vertical, contingente y no institucionalizada desde la empresa hacia el trabajador, permitirá evidenciar la necesidad de exigir mayores niveles de protección amparados en la legalidad existente. Es decir, despersonalizar las relaciones entre trabajador individual y patrón, y poner en su lugar negociaciones más institucionalizadas entre organizaciones de trabajadores y empresa, permitiría manifestar de manera más segura el malestar subjetivo individual por la vía de una reivindicación colectiva. Las trabas para esto se encuentran en los otros dos factores ya señalados (las bajas expectativas salariales y laborales por las escasas oportunidades que ofrece el mercado de trabajo en el territorio inmediato, y el miedo a las represalias de la empresa, incluyendo el eventual despido) que conforman un círculo vicioso propio de las particularidades que aún persisten en la ruralidad y que en gran medida explican, precisamente, el mayor retraso organizacional que se observa en este sector de los trabajadores en comparación con las zonas urbanas.

\section{Agradecimientos}

Esta investigación no hubiera sido posible sin el financiamiento de la Dirección de Posgrado de la Facultad de Ciencias Sociales de la Universidad de Chile. Apreciamos también la participación en el equipo de investigación de David Ávalos y Fernanda Azócar, así como la retroalimentación y acogida institucional del Centro de Investigación Político Social del Trabajo (CIPSTRA). Finalmente se agradece la confianza de cada uno de los entrevistados de las cuatro empresas analizadas, y sinceramente esperamos que estos resultados sean útiles para sus luchas por mejores condiciones de trabajo y vida en el campo chileno. 


\section{Notas}

1 Datos del “Anuario Estadístico 2014” de la Dirección del Trabajo. La tasa de sindicalización presentada corresponde al cociente entre fuerza de trabajo afiliada y fuerza de trabajo ocupada, descontando a los asalariados del sector público, imposibilitados de organizarse en sindicatos.

$\underline{2}$ El estudio se centró en los componentes subjetivos de la precariedad laboral como factor para la explicación de la conflictividad, asumiendo que también existen otras causas históricas y sobretodo legales que dificultan la organización de los trabajadores. Al respecto, ver (Gómez, 2002).

$\underline{3}$ Esta distinción se realizó a partir de los datos de la encuesta de Caracterización Socioeconómica Nacional 2011.

4 En Chile, la existencia de contrato de trabajo y el carácter indefinido de este, respectivamente, permiten acceder a beneficios de seguridad social como cobertura de salud, cotizaciones previsionales para el fondo de pensión y seguro de cesantía.

$\underline{5} \mathrm{Al}$ respecto, cabe destacar que la dispersión del puntaje en el índice de protección del empleo entre quienes se encuentran insatisfechos es bastante mayor que entre quienes se encuentran satisfechos, lo que sugiere que quienes se encuentran satisfechos suelen ser un grupo más específico de trabajadores (no necesariamente los menos precarizados en términos objetivos) que lo que se encuentran insatisfechos.

\section{Bibliografía}

Bayón, M. C. (2006). Precariedad social en México y Argentina: Tendencias, expresiones y trayectorias nacionales. Revista de la CEPAL 88, 133-152.

Bendini, M. (2008). Actores sociales y reestructuraciones de los ámbitos rurales y agrarios de América Latina. En E. Pérez, M. Farah, y H. C. de Grammont, La nueva ruralidad en América Latina. Avances teóricos y evidencias empíricas (pp. 133-150). Bogotá: Pontificia Universidad Javeriana.

Bengoa, J. (1983). El Campesinado Chileno después de la Reforma Agraria. Santiago de Chile: Ediciones SUR.

Bengoa, J. (1988). Historia social de la agricultura chilena. Tomo I: El poder y la subordinación. Santiago de Chile: Ediciones Sur.

Betancor, A., Modrego, F., \& Berdegué, J. (2008). Crecimiento Agrícola y Pobreza Rural en Chile y sus Regiones. Santiago de Chile: RIMISP.

C. de Grammont, H. (2004). La Nueva Ruralidad en América Latina. Revista Mexicana de Sociología 66, 279-300.

Canales, M. (2005). La nueva ruralidad en Chile. Apuntes sobre subjetividad y territorios vividos. Chile Rural, un desafío para el Desarrollo Humano, 33-40.

Canales, M., \& Canales, A. (julio de 2012). La Nueva Provincia: (Re)poblamiento de los territorios agrarios. Chile 1982-2002. Revista Anales 3, 157-173.

Carámbula, M. (2009). Movilidad espacial y precariedad laboral en los trabajadores rurales temporales: el caso de los equiladores de Villa Sara (Tesis Magíster en Ciencias Agrarias, opción Ciencias Sociales). Facultad de Agronomía, UdelaR, Montevideo. 
Caro, P., \& de la Cruz, C. (2005). Contratistas e Intermediación Laboral en la Agricultura de exportación. Santiago: CEDEM.

Castel, R. (1997). La metamorfosis de la Cuestión Social. Buenos Aires: Paidós.

Castel, R. (2006). La Inseguridad Social: ¿Qué es estar protegido? Buenos Aires: El Manantial.

Castel, R. (2010). El ascenso de las incertidumbres. Trabajo, protecciones, estatuto del individuo. Buenos Aires: Fondo de Cultura Económica.

Cid, B. (2001). Trabajo temporeras de la Agroindustria. Núcleo de contradicción en el Nuevo Mundo Rural. Desafio a las Políticas Públicas. Santiago: Documento $\mathrm{N}^{\circ} 6$. Debates y reflexiones para la investigación social. PREDES.

Dávila, A. (1995). Las perpectivas metodológicas cualitativa y cuantitativa en las ciencias sociales. Debate teórico e implicaciones praxeológicas. En J. M. Delgado, \& J. Gutiérrez, Métodos y técnicas cualitativas de investigación en ciencias socielas (pp. 69-86). Madrid: Síntesis.

ESOPE. (2005). Precarious Employment in Europe. A Comparative Study of Labour Market Related Risks in Flexible Economics. Bruselas: European Commission.

Fundación SOL. (2015). Informe Mensual de Calidad del Empleo (IMCE). Diciembre 2014 - Febrero 2015. Santiago de Chile: Fundación SOL.

Gaillie, D., \& Paugam, S. (2000). Welfare Regimes and the Experience of Unemploymet in Europe. Nueva York: Oxford University Press.

Glaser, B. (1992). Basic of Grounded Theory Analysis: Emergence vs. Forcing. Mill Valley: Sociology Press.

Glaser, B., \& Strauss, A. (1967). The discovery of Grounded Theory: Strategies for Qualitative Research. New York: Aldine Publishing.

Gómez, S. (2002). Organización campesina en Chile: Reflexiones sobre su debilidad actual. Revista Austral de Ciencias Sociales 6, 3-18.

Gómez, S. (2011). Reflexiones sobre la nueva ruralidad en América Latina. Revista Paraguaya de Sociología 48(138), 57-78.

Gómez, S., \& Klein, E. (1993). Los pobres del campo. El trabajador eventual. Santiago de Chile: FLACSO.

Guadarrama, R., Hualde, A., \& López, S. (2012). Precariedad laboral y heterogeneidad ocupacional: una propuesta teórico-metodológica. Revista Mexicana de Sociología, 213-243.

Haesbaert, R. (2011). El mito de la desterritorialización. Del "fin de los territorios" a la multiterritorialidad. Ciudad de México: Siglo XXI Editores.

Menéndez, V. (2009). Trabajadores Forestales. Hacia una mirada a su realidad desde la precariedad laboral. (Trabajo Final de Grado. Licenciatura de Sociología.) Facultad de Ciencias Sociales, UdelaR, Montevideo.

Muñoz, E., \& Navarro, P. (2011). Análisis del déficit hídrico en la Agricultura de la Región del Maule, Chile. Revista Interamericana de Ambiente y Turismo, 7(1), 25-32.

Narbona, K., Páez, A., \& Tonelli, P. (2011). Precariedad Laboral y Modelo Productivo en Chile. Fundación SOL. Santiago de Chile: Ideas para el Buen Vivir. Recuperado el 20 de Diciembre de 2015, de http://www.fundacionsol.cl/estudios/precariedad-laboral-y-modelo-productivo-en-chile/ 
ODEPA. (2009). Valor de la tierra agrícola y sus factores determinantes. Santiago de Chile: Ministerio de Agricultura.

ODEPA. (2014). Región del Maule. Información regional 2014. Santiago de Chile: Ministerio de Agricultura.

Paugam, S. (1991). La disqualification sociale. Essai sur la nouvelle pauvreté. París: PUF.

Paugam, S. (2000). Le Salarié de la Precarité: les nouvelles formes de l'integration profesionelle. París: Presses Universitaires de France.

Paugam, S. (Septiembre de 2012). Protección y reconocimiento. Por una sociología de los vínculos sociales. Papeles del CEIC 82.

Paugam, S., \& Zhou, Y. (2007). Job insecurity. En D. Gallie, Employment, Regimes and Quality of Work. Nueva York: Oxford University Press.

Pérez Sáinz, J. P. (2002-2003). Exclusión laboral en América Latina: viejas y nuevas tendencias. Sociología del Trabajo 47, 107-138.

Pérez, D., Medel, R., \& Velásquez, D. (2015). El desarrollo de la huelga ilegal en el Chile contemporáneo (1990-2009). Latin American Studies Association Congress. Recuperado el 18 de Diciembre de 2015, de http://http://coes.cl/wp-content/uploads/2015/01/Art\%C3\%ADculo-OHL-COES-LASA-20151.pdf

Pérez, E., Farah, M., \& C. de Grammont, H. (2008). Introducción. En E. Pérez, M. A. Farah, \& H. C. de Grammont, La nueva ruralidad en América Latina. Avances teóricos y evidencias empíricas (pp. 13-18). Bogotá: Pontificia Universidad Javeriana.

Piñeiro, D. (2008). El Trabajo Precario en el Campo Uruguayo. Montevideo: Comisión Sectorial de Investigación Científica. Facultad de Ciencias Sociales. Universidad de la República.

Piñeiro, D. (2011). Precariedad objetiva y subjetiva en el trabajo rural: Nuevas evidencias. Revista de Ciencias Sociales, 24(28), 11-33.

PNUD. (2008). Desarrollo Humano en Chile Rural. Santiago de Chile: PNUD.

Reardon, T., Berdegué, J., \& Escobar, G. (2004). Empleo e ingresos rurales no agrícolas en América Latina: síntesis e implicaciones de políticas. En C. -F. RIMISP, Empleo e ingresos rurales no agrícolas en América Latina (pp. 15-34). Santiago de Chile: CEPAL.

Riella, A., \& Mascheroni, P. (2009). Explorando la calidad del empleo en la forestación. Un estudio de caso. Montevideo: Comisión Sectorial de Investigación Científica. Facultad de Ciencias Sociales. Universidad de la República y Programa ALFA.

Rodgers, G., \& Rodgers, J. (1989). Precarios Jobs in Labour Market Regulation. The Growth of Atypical Employment in Western Europe. Génova: International Institute for Labour Studies / Free University of Brussels / International Labour Organization.

Sack, R. (1986). Human territoriality: its theory and history.Cambridge University Press 